\title{
Représentations de l'atome et visualisations de la réalité moléculaire
}

\section{Charlotte Bigg}

\section{(2) OpenEdition \\ 1 Journals}

Édition électronique

URL : https://journals.openedition.org/rbnu/2694

DOI : $10.4000 /$ rbnu. 2694

ISSN : 2679-6104

Éditeur

Bibliothèque nationale et universitaire de Strasbourg

\section{Édition imprimée}

Date de publication : 1 novembre 2012

Pagination : 32-41

ISSN : 2109-2761

\section{Référence électronique}

Charlotte Bigg, «Représentations de l'atome et visualisations de la réalité moléculaire », La Revue de la BNU [En ligne], 6 | 2012, mis en ligne le 01 novembre 2012, consulté le 08 août 2021. URL : http:// journals.openedition.org/rbnu/2694; DOI : https://doi.org/10.4000/rbnu.2694

\section{(c) (i) (9)}

La Revue de la BNU est mise à disposition selon les termes de la Licence Creative Commons Attribution - Pas d'Utilisation Commerciale - Partage dans les Mêmes Conditions 4.0 International. 

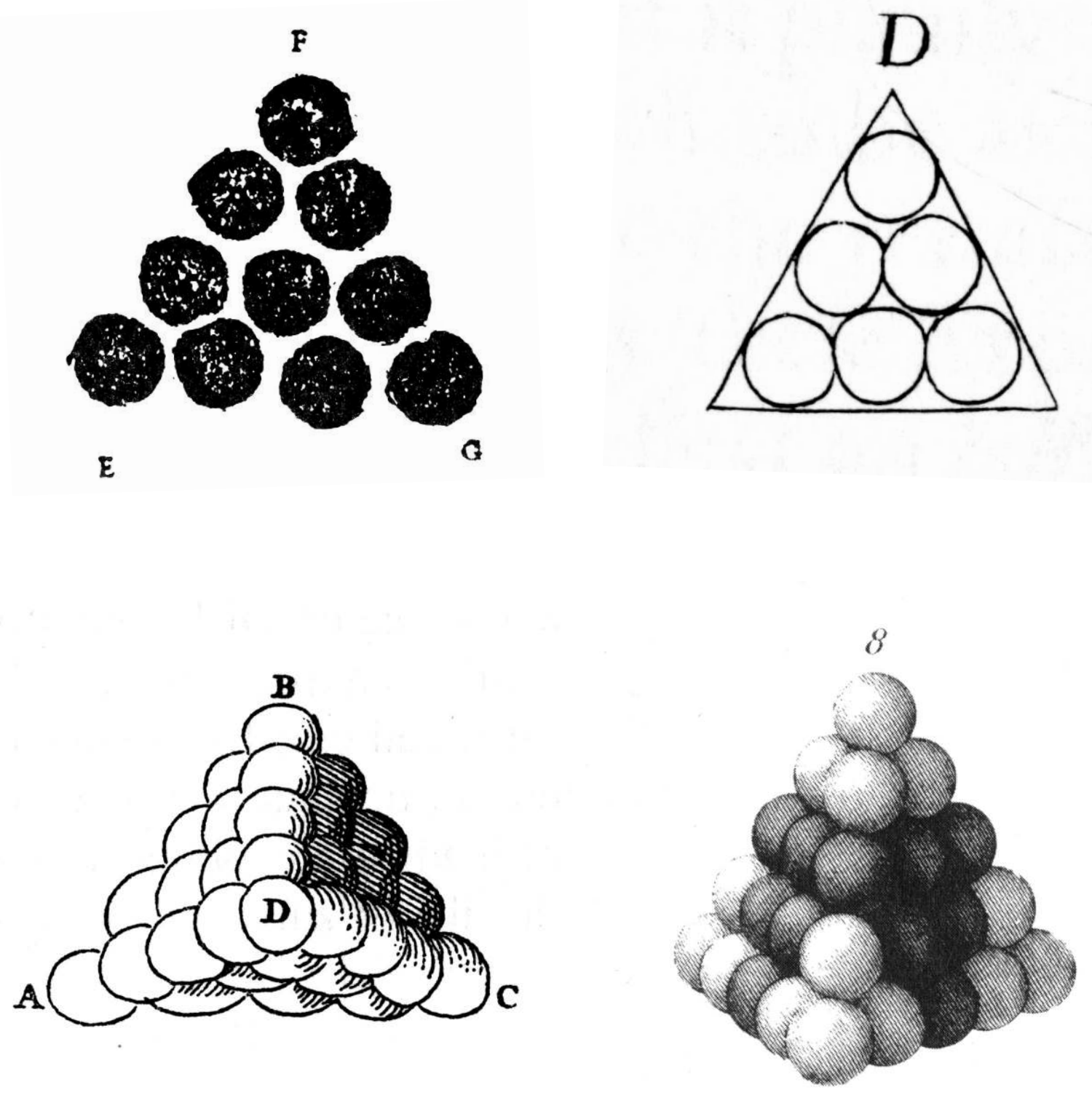

L’atome-sphère, une tradition iconographique depuis le $16^{\mathrm{e}}$ siècle. De g. à d. et de h. en b. : Giordano Bruno, De triplici minimo et mensura, Francfort sur le Main, 1591, p. 90 ; Robert Hooke, Micrographia, Londres, 1665, p. 82 ; Christian Huygens, Traité de la lumière, Leyde, 169o, p. 519 ; William Hyde Wollaston, On the elementary particles of certain crystals, in Philosophical Transactions, vol. 103 (1813), p. 51-63, tableau II, p. 62. Illustrations tirées de Christoph Lüthy, The invention of atomist iconography, in Wolfgang Lefevre, Jürgen Renn, Urs Schöpflin (éd.) :

The power of images in early modern science, Bâle, 2003, p. 117-138. 


\section{Représentations de l'atome et visualisations de la réalité moléculaire}

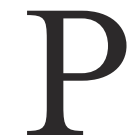

our aborder la question de la représentation des savoirs, quel meilleur sujet choisir que celui de l'atome ? Entité invisible par excellence, dont l'existence même fit longtemps débat sans que cela ne l'empêche de faire l'objet de représentations visuelles multiples et variées, l'atome suggère qu'une image est bien plus qu'une simple illustration : le travail de mise en image participe de l'étude scientifique des objets. En sciences aussi, la représentation n'est pas donnée mais construite, et son aboutissement, l'image, façonne à son tour la compréhension des phénomènes ainsi matérialisés et imprègne nos imaginaires ${ }^{1}$.

Si l'on doit le terme d'atome au grec ancien (atomos, " indivisible "), la conception d'un constituant ultime de la matière remonte probablement plus loin encore. Mais ce sera seulement à la fin du $16^{\text {e }}$ siècle, plus précisément en 1591, avec le De triplici minimo et mensura de Giordano Bruno, que se met en place une convention de représentation qui perdure jusqu'à nos jours, dépeignant l'atome sous la forme d'une petite sphère (voir illustration p. 32). Cette continuité visuelle suggère l'existence d'une logique iconographique propre, au-delà des divergences très importantes dans les conceptions de la constitution de la matière et de ce que recouvre le terme d'atome ${ }^{2}$.

L'atome fut longtemps une construction de l'esprit philosophique, voire plus tard entre les mains des chimistes un modèle heuristique et pédagogique permettant de penser les transformations chimiques

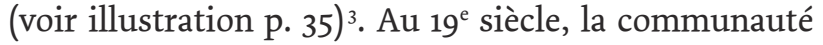
savante s'enflamme autour de la question de la réalité empirique de l'atome. Le débat entre " continuistes" et " discontinuistes " opposés dans leur conception de la nature ultime de la matière (énergie ou particules) sera résolu sur fond de découvertes expérimentales qui dans un premier temps fourniront des armes à l'un ou l'autre camp, avant de profondément transformer les conceptions de la matière et de rendre caducs ces débats.

Le tournant $\mathrm{du} 2 \mathrm{O}^{\mathrm{e}}$ siècle révèle en effet l'existence d'un monde insoupçonné, peuplé de particules et de rayons rendus perceptibles grâce à de nouveaux dispositifs ingénieux : cliquetis des compteurs Geiger ou scintillement d'écrans de sulfure de zinc comptabilisent le passage de particules et rayons ionisants, les chambres à brouillard révèlent la trajectoire de particules tel le sillage d'un avion dans le ciel, tandis que la lumière obscure des rayons $\mathrm{X}$ ou radioactifs laisse son empreinte dans les émulsions photographiques. Ces traces éphémères sont souvent capturées sous la forme de photographies qui sont à la fois le révélateur, le moyen d'étude et la preuve tangible de ces rayonnements. Diffusées auprès des collègues, imprimées dans les journaux savants et populaires, elles constituent pour leurs auteurs les garants de leur priorité dans la découverte (voir illustration p. 37).

Ces clichés, dont rapidement l'apparence s'uniformise, forment un nouvel ensemble d'images expérimentales du monde subatomique qui prennent leur place à côté de l'atome sphérique sans jamais le supplanter, et qui à leur tour contribuent à l'élaboration de nouveaux modèles de l'atome ; à commencer par l'atome de Bohr "planétaire ", autre icône visuelle qui continue de peupler notre imaginaire malgré sa remise en cause par les physiciens dès les années $1920^{4}$.

On entrevoit déjà les différentes fonctions que peuvent remplir les représentations visuelles dans ce contexte, 
à la fois outils de recherche, techniques de visualisation, traces instrumentales, preuves enfin de l'existence empirique de nouvelles entités invisibles, mais aussi moyen de communication et de vulgarisation des résultats scientifiques. On aurait tort d'ailleurs de trop vouloir distinguer ce qui relève de la recherche " en propre " et de sa communication. La science est une entreprise collective, qui passe le plus souvent par une circulation intense des personnes, des instruments, des informations au sein des milieux scientifiques et au-delà. La communication par des moyens verbaux, écrits ou visuels est à ce titre le cœur du travail scientifique et non pas simplement son prolongement.

Les travaux du physicochimiste parisien Jean Perrin en fournissent un exemple particulièrement frappant. Ayant débuté sa carrière par des travaux expérimentaux montrant que les rayons cathodiques sont constitués de particules porteuses de charges négatives (que l'on baptisera ultérieurement électrons), il se distingue surtout dans les années 1900 par une série d'expériences sur le mouvement brownien destinées à apporter la preuve décisive de l'existence empirique de l'atome.

L'importance que revêt l'image pour Perrin est manifeste dès les premières pages d'un long article publié dans les Annales de chimie et de physique en 1909, la synthèse la plus complète de ses recherches sur ce sujet. Après une présentation succincte du mouvement brownien, cette agitation incessante et désordonnée que l'on observe au microscope lorsque l'on saupoudre des particules très fines sur un liquide au repos, Perrin indique que l'" on peut rendre ce phénomène visible à tout un auditoire mais [que] cette projection est difficile ». Il consacre ensuite plusieurs pages à en détailler la technique en indiquant toutes les précautions à prendre : passage des rayons lumineux à travers une cuve d'eau pour filtrer les rayons calorifiques susceptibles de réchauffer la solution, usage d'un objectif à immersion, d'un oculaire à fort grossissement et d'un prisme à réflexion totale, qui projettent une image de l'agitation des granules sur un écran de verre dépoli et quadrillé ; enfin préparation d'une émulsion appropriée, avec des granules de taille suffisante pour être perceptibles par des spectateurs situés à plus de trois mètres de distance ${ }^{5}$ (voir illustration p. 37). L'expertise en la matière de Perrin, comme il apparaît plus loin dans le texte, provient de son usage, dans ses expériences mêmes, de ce dispositif de projection, notamment pour mesurer le déplacement de particules individuelles (voir illustration p. 39).

On voit l'utilité d'un tel appareillage pour le dénombrement de particules ou l'étude du mouvement de particules individuelles; mais son utilisation délibérée lors de conférences scientifiques ou destinées au grand public est peut-être plus surprenante. Ce dispositif joue en effet un rôle non négligeable dans la démonstration du physicochimiste, dont l'argumentaire repose sur une analogie entre le mouvement brownien de particules visibles et l'agitation moléculaire par définition imperceptible. Comme le ballottement d'une bouée par les vagues reste perceptible à une grande distance, même lorsque les vagues sont devenues invisibles, le mouvement brownien des particules en suspension est une conséquence directe et témoigne, à notre échelle, de l'agitation moléculaire. Par le truchement du mouvement brownien, l'agitation moléculaire est suggérée à la vue. "Ainsi ", écrit Perrin, " les granules en suspension fonctionnent comme des molécules visibles d'un gaz parfait $"{ }^{6}$.

Le rôle de la perception sensible dans le travail scientifique avait été théorisé quelques années auparavant par le savant dans son ouvrage programmatique Traité de chimie physique. Les principes de 1903. Il y explique vouloir surmonter l'opposition entre atomistes et anti-atomistes, et réconcilier les méthodes inductive (caractérisée par le " dédain de tout ce qui ne peut se réduire à de la sensation effectivement réalisable ") et déductive (" qui consiste à imaginer a priori pour la matière une structure dont la perception directe échappe encore à nos sens imparfaits ") en démontrant que l'atome est plus qu'une simple hypothèse : 
the solid arm of the former into the tubular arm of the latter, we have thas construeted a molenile of hydrochlorie acid. The attraction units of the two elements are saturated; we can add no more; the building is finished.

Fin. 7.

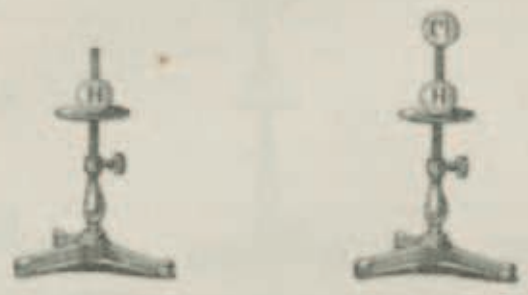

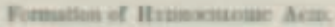

The next hydrogen atom we combine in a similar manner with an ntom of oxygnt, but no closed moleenle is thas produced. One of the attrnetion units of the oxygen is not yet saturated, as ibdieated by the one arw rumaining uneovered. Only by fixing upon this arm a second hydrogen atom we saturate this second attraction unit also. The elosed water molecule is a finished building.

Fra. a.
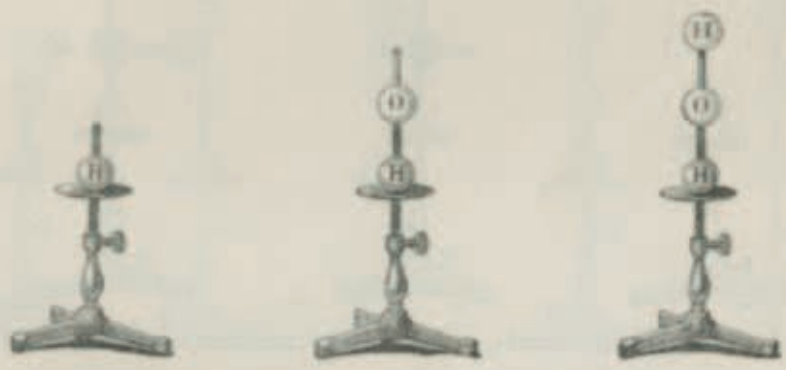

Romather of Waris

Again, an atom of nitrogen is inserted into tlie hydrogen atom on our third stand ; the two nitrogen arms which are left expoed suffieiently indicate that two attraetion units remain unsaturated, and have uceordingly to be provided with two atoms of an univalent element.

Vol. IV. (No. 42.)

L'atome-sphère sous forme de modèle en trois dimensions, permettant de visualiser les formules chimiques. Développé en 1865 par le chimiste August Wilhelm Hofmann pour ses conférences publiques, il s'agit à l'origine de balles de croquet peintes reliées par des tubes de métal. August Wilhelm Hofmann, On the combining power of atoms, in Proceedings of the Royal Institution of Great Britain, vol. 4, 1865, p. 417 
" Mais il me paraît qu'on a encore le droit d'attribuer aux molécules, atomes ou corpuscules une réalité plus grande (que celle du modèle). Et je ne retombe pas dans la métaphysique. Je ne cesse pas d'oublier que la sensation est la seule réalité. C'est la seule réalité, à condition d'adjoindre aux sensations actuelles toutes les sensations possibles".

Perrin s'attachera dès lors à rechercher " une conception attribuant à la matière une structure telle que ses propriétés sensibles en puissent être aisément déduites $" 7$.

On comprend alors l'intérêt de la projection, qui permettait à Perrin lors de sa conférence de transformer la signification du phénomène sous les yeux mêmes de son public. Au cours de sa démonstration, le mouvement brownien des granules sur l'écran devenait le reflet, la matérialisation perceptible de l'agitation incessante et désordonnée des molécules dans le liquide : " Bref, le seul examen du mouvement brownien pouvait suffire à suggérer que tout fluide est formé de molécules élastiques, animées d'un mouvement éternel " 8 .

Associé à un travail important de mesure et de calcul pour obtenir une détermination numérique du nombre d'Avogadro et des dimensions moléculaires, cet argumentaire emporta l'adhésion d'une grande partie des scientifiques. La composante visuelle fut particulièrement remarquée par certains observateurs contemporains : en témoigne la rumeur, inexacte mais révélatrice, qui circula à l'époque que Perrin avait développé " une méthode d'isoler et de voir les atomes " ${ }^{9}$. C'est l'une des stratégies adoptées par Perrin pour faire glisser le débat sur l'existence des atomes du terrain philosophique et théorique vers un terrain plus expérimental. On notera que Perrin entend par là reconfigurer la conception de l'atome tout en ayant à cœur de se situer rhétoriquement et visuellement dans le prolongement de la tradition atomiste remontant à l'Antiquité, y compris par la référence visuelle à l'iconographie de l'atome sphérique (voir illustration p. 37).

A partir de 1911, Perrin remplacera la projection, toujours délicate à réaliser, par la projection de films du mouvement brownien dans ses conférences, pour lesquels il fera régulièrement appel à Jean Comandon, pionnier du cinéma scientifique et de la cinématographie microscopique. De fait, la cinématographie du mouvement brownien constitue l'une des premières tentatives de cinéma scientifique, expérimentée dès
1908 par Victor Henri au Collège de France dans l'ancien laboratoire d'Etienne-Jules Marey, inventeur d'une technique anticipatrice du cinéma, la chronophotographie $^{10}$; recherches dont non seulement Comandon, mais aussi Jean Perrin avaient connaissance puisque ce dernier adoptera le style de visualisation " chronophotographique " du mouvement brownien de particules individuelles développé par V. Henri (voir illustration p. 39).

Comme la projection et selon des modalités technologiques assez proches, le cinéma est à la fois un instrument de recherche et un moyen de communication, d'autant plus qu'il reste encore une technique expérimentale, a fortiori lorsqu'il est microscopique, ralenti, etc. Le cinéma, vanté dès ses débuts pour sa capacité à représenter le mouvement d'une manière particulièrement vivante, est une option technologique qui sert particulièrement bien les ambitions de Jean Perrin.

Remarquons que l'usage du cinéma en science n'allait pas de soi à cette époque : lors des premières projections filmées à l'Académie des sciences, de nombreux savants exprimèrent des réticences à l'encontre de ce nouveau média qui fleurait trop la fête foraine et dont la fiabilité restait à démontrer. Les frontières entre film scientifique et de divertissement étaient (et restent parfois) assez floues, puisqu'à l'issue de son doctorat en 1909 Jean Comandon sera d'abord recruté par Charles Pathé. C'est dans son nouveau laboratoire de microcinématographie de l'usine Pathé de Vincennes que Comandon réalisera des films à destination des scientifiques et du grand public. Le film sur le mouvement brownien réalisé pour Perrin est luimême inclus dans le programme Pathé dès 1911.

Comandon, Perrin et d'autres se feront les avocats infatigables d'un emploi sérieux du film cinématographique, en s'élevant contre des usages par trop profanes de leurs productions (par exemple la diffusion dans des lieux d'amusement de films spectaculaires d'opérations chirurgicales à l'origine destinés à l'enseignement médical) et en s'impliquant dans la réalisation de films pédagogiques. Après la Première Guerre mondiale, Comandon réalise notamment pour Pathé un grand nombre de films scientifiques de propagande hygiéniste qui sont largement diffusés ${ }^{11}$.

Au-delà de ses usages expérimentaux, Perrin comme Comandon conçoivent le cinéma comme un outil pédagogique, reprenant une rhétorique utilisée auparavant pour promouvoir la projection. Le cinéma 


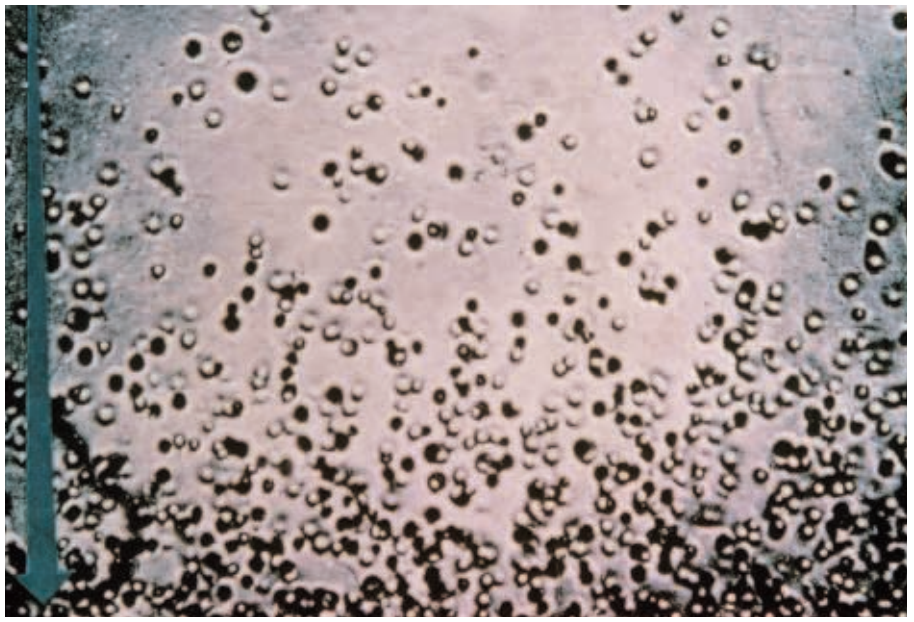

Microphotographie de la répartition en hauteur de sphérules de résine en suspension dans l'eau. Photographie reproduisant un cliché de Perrin, réalisée par le palais de la Découverte pour la présentation de ses expériences sur le mouvement brownien.

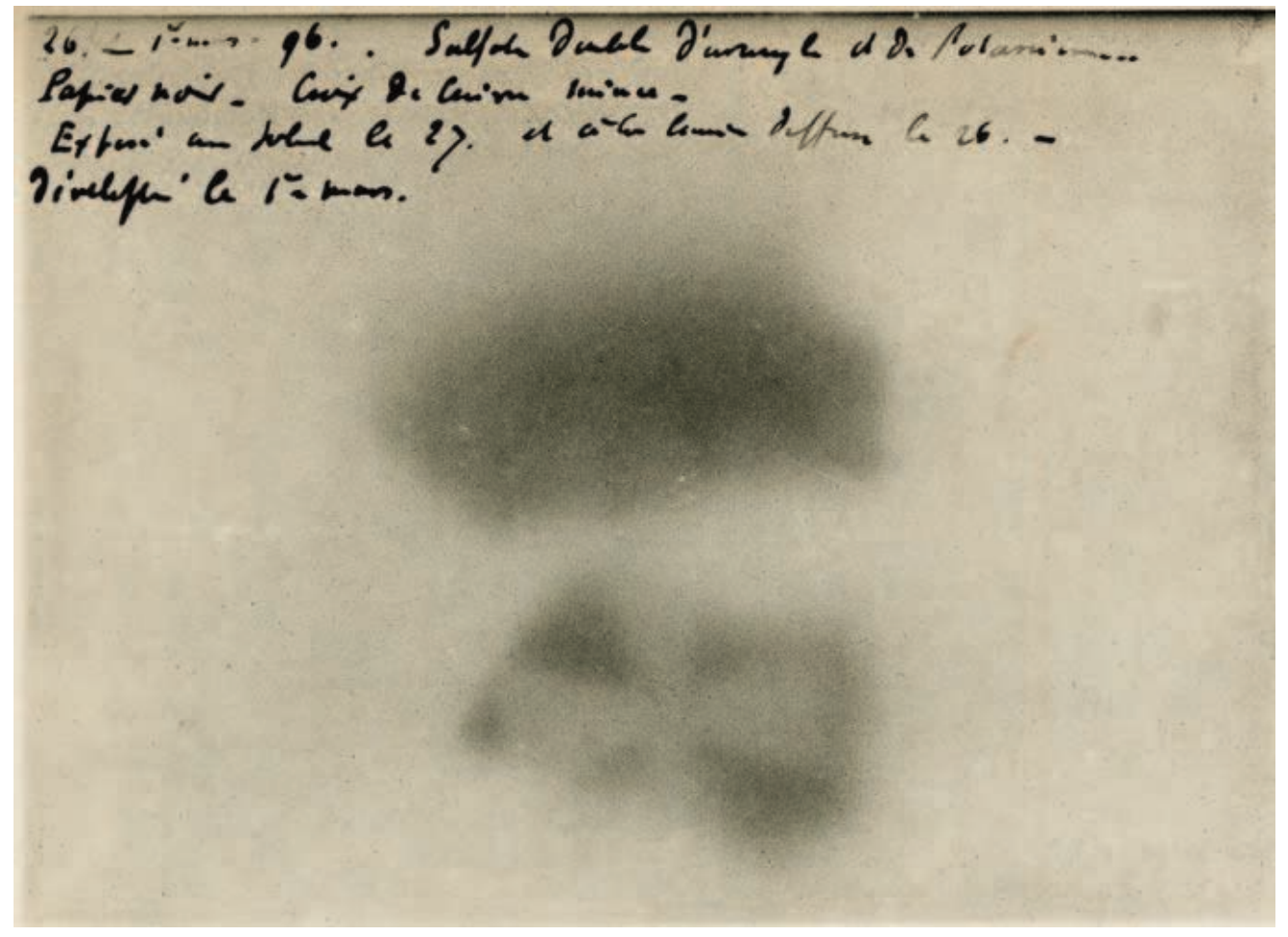

La trace photographique comme preuve de la découverte de la radioactivité.

Photographie réalisée par Henri Becquerel en 1896. Henri Becquerel, Recherches sur une propriéte nouvelle de la matière,

in Mémoires de l'Académie des sciences de l'Institut de France, Paris, 1903, fig. 1 

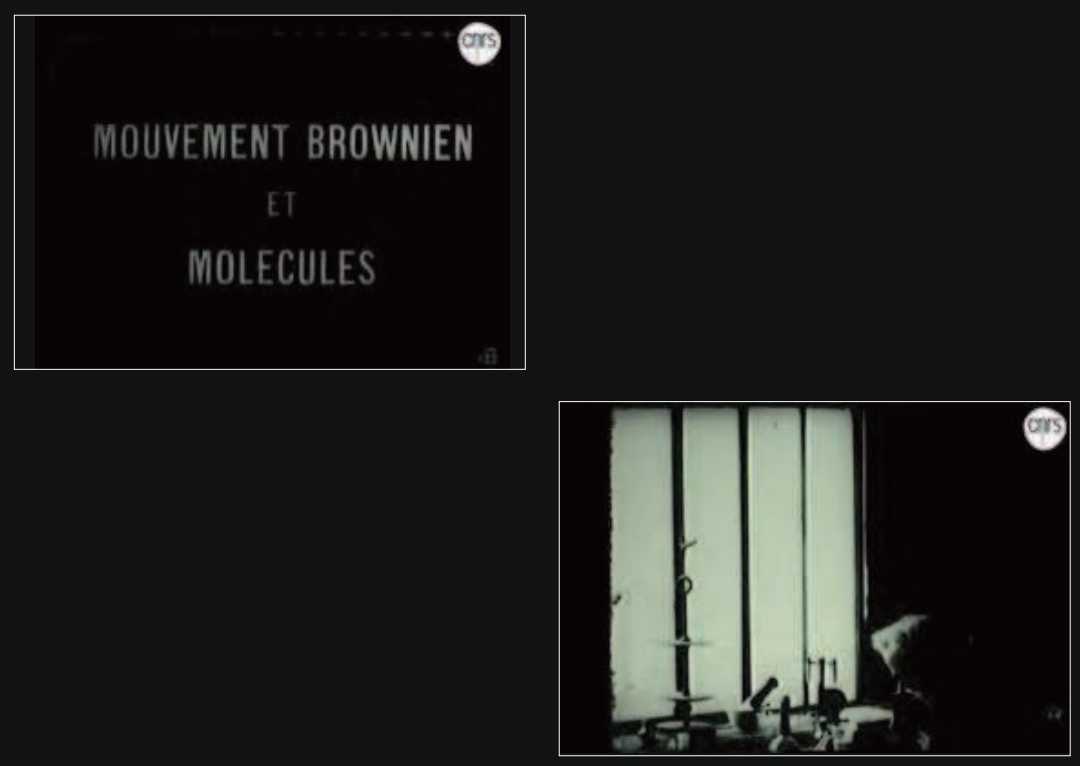

Ainsi les particules en sus pension, comme feraient de bouees indicatrices, et d'au tant plus fidèlement qu'ellesont plus petites, révèlen une agitation interne violent et désordonnèe dans ce qu nous appelons un fluide eI equilibre

ars

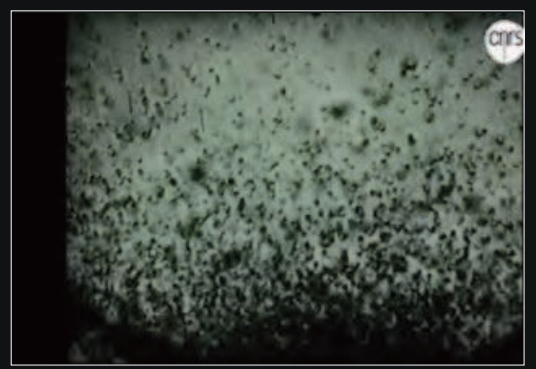

Mouvements browniens et molécules. Plans d'un film réalisé en 1921 par Jean Perrin L'agitation interne ains constatée s'accorde avec it vieille hypothèse des atomis tes grees, suivant laquell, toute matière est faite di molécules imperceptibles, qu s'agitent et se heurtent san: resse au hasard, conservan éternellement leur mouve mant

Sil laut s'élever de 5 milliemes de millimetres dans l'émulsion ef de 5 kilomètres dons l'atmosphere pour observer une méme rorélac. lion, c'est que les molécules d'oir sont 1 milliard de lois plus légères que les granules de l'émulsion 


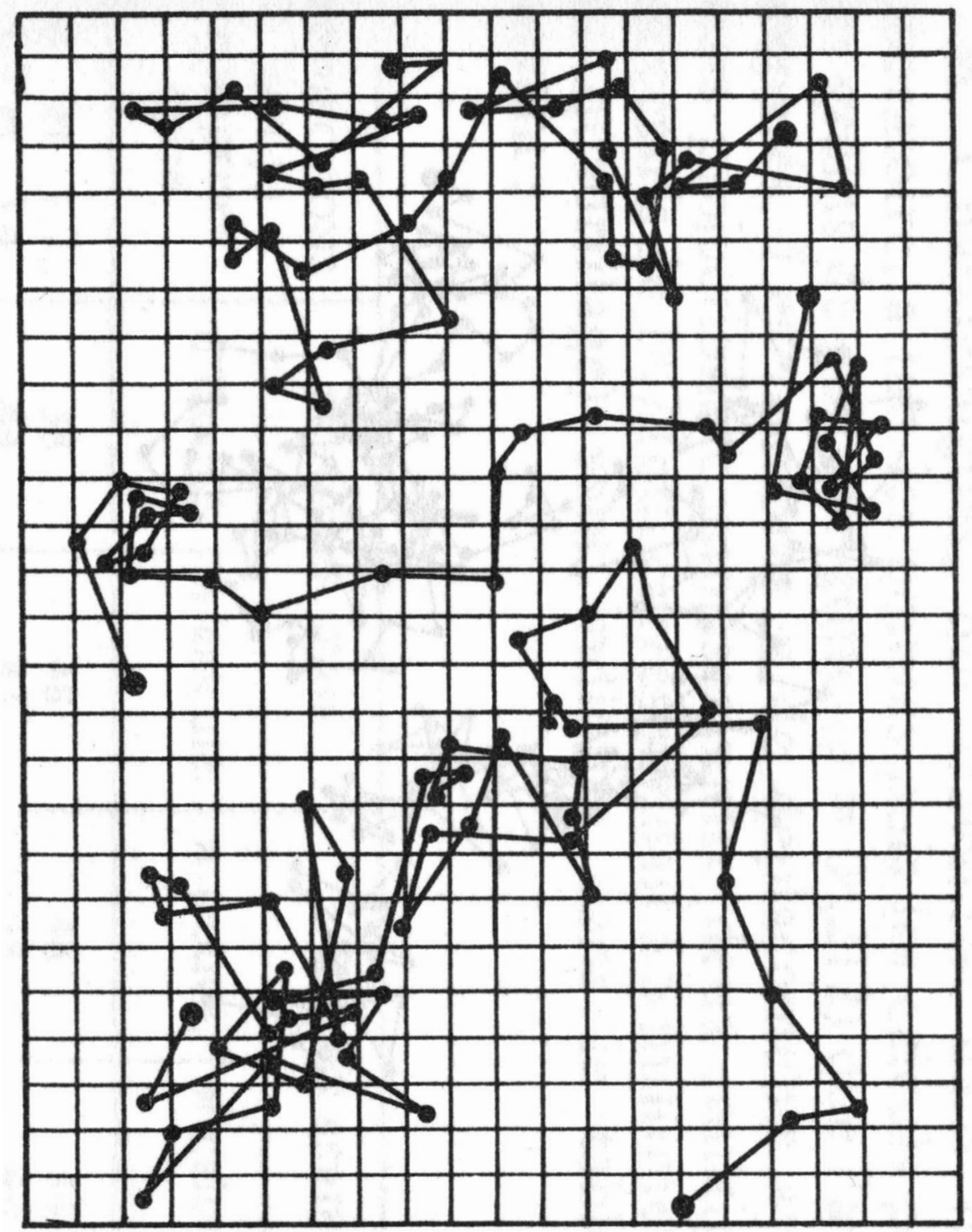

Dessin représentant le mouvement brownien de trois particules distinctes. Au moyen d'un projecteur, l'image de la solution est projetée sur un écran dépoli et quadrillé. A intervalles réguliers, les positions successives d'une particule sont notées par des points, ce qui permet de calculer le déplacement moyen des particules. Jean Perrin, Mouvement brownien et réalité moléculaire, in Annales de chimie et de physique, sér. 8, vol. 18, 1909, p. 81 
est intégré à une conception réformiste de l'enseignement qui privilégie la leçon de choses, le contact avec les objets et la perception sensible, à l'encontre de l'enseignement purement verbal et supposé aride ${ }^{12}$. Le rapport pour 1920 de la Commission extra-parlementaire chargée d'étudier les moyens de généraliser l'application du cinématographe dans les différentes branches de l'enseignement (que Comandon rejoindra peu après) formule cette attitude de la manière suivante : " l'intérêt du spectacle sollicite l'attention des élèves, de ceux mêmes dont l'imagination est paresseuse. Les souvenirs qui leur restent d'une image animée sont plus nets et persistent plus longtemps. Admirable auxiliaire pour le maître dont il vivifie l'enseignement, le cinématographe permet de réduire le verbalisme qui délaie et affaiblit l'idée : il met sous les yeux la synthèse vivante des êtres et des choses. 'Voir c'est presque savoir', écrit justement notre distingué collègue $\mathrm{M}$. Roux. Il ne se borne pas à donner la vision rapide du mouvement et de l'action, il les ralentit, les décompose et par là même devient un merveilleux instrument de démonstration et d'analyse (...) Quand l'observation directe de la réalité n'est pas possible, les projections cinématographiques y suppléent. Elles sont le document précieux, le témoignage réel qui confirme la leçon du maître $"{ }^{13}$.

Exploitant le fort impact visuel de la projection et du cinéma, Perrin utilise ces technologies pour emporter l'adhésion de ses publics dans sa démonstration de la réalité moléculaire. Ces dispositifs permettent littéralement au physico-chimiste de poser la réalité moléculaire comme une "sensation possible ". Ils témoignent d'une grande cohérence entre son approche scientifique et ses conceptions pédagogiques, qui reposent sur la perception sensorielle comme élément essentiel de la connaissance.

Ces convictions continueront de guider les activités de Perrin, à la fois dans ses recherches sur d'autres phénomènes permettant de visualiser la discontinuité de la matière (couches monomoléculaires) et dans ses interventions publiques. Après la Première Guerre mondiale et plus particulièrement après l'attribution en 1926 du prix Nobel de physique pour ses recherches sur le mouvement brownien, Perrin sera amené lors d'innombrables conférences à présenter et représenter ses travaux, sollicitant régulièrement Comandon pour de nouvelles copies des films du mouvement brownien, allant jusqu'à produire en 1921 un film où il se met lui-même en scène, répétant ses désormais célèbres expériences (voir illustration p. 38). A travers ce film (et d'autres films comparables réalisés à la même époque) et l'usage revendiqué de la technologie moderne du cinéma, est proposée une nouvelle manière de représenter visuellement non seulement les objets scientifiques et la recherche, mais aussi la figure du scientifique, et ce au moment où Perrin met à profit cette présence médiatique pour promouvoir la recherche et son financement public.

On connaît le rôle qu'a joué Perrin par la suite dans la structuration de la recherche en France, notamment en sa qualité de sous-secrétaire de la recherche scientifique en 1936-1937. Dans les deux grandes institutions qu'il contribua à mettre sur pied, le CNRS et le palais de la Découverte, on retrouve de nombreux éléments de l'approche caractéristique de Perrin telle que nous l'avons brièvement esquissée, et qui persistent encore aujourd'hui.

Le palais de la Découverte, le volet scientifique de l'Exposition internationale des arts et techniques dans la vie moderne tenue à Paris en 1937, pérennisé en 1938 pour devenir le premier grand musée des sciences en France, incarne ainsi la conception de la pédagogie défendue par Perrin, y compris dans ses choix technologiques. Au moment de son inauguration, il affirme :

« Dans ce palais, nous avons voulu réaliser une exposition vivante où sont autant que possible répétées de façon spectaculaire, avec les ressources les plus modernes, les découvertes fondamentales qui ont élargi notre intelligence (...) Les expériences sont refaites sous les yeux des visiteurs par des " démonstrateurs " qui les expliquent, s'aidant au besoin de phonogrammes synchronisés ou de films cinématographiques ${ }^{14}$.

Perrin fera d'ailleurs consacrer une salle de la section de physique à la " Discontinuité de la matière et réalité moléculaire ", dans laquelle ses propres expériences sont mises en scène, y compris à l'aide de projections et de films du mouvement brownien, lui permettant de s'inscrire matériellement dans l'histoire des sciences telle qu'elle est communiquée au palais. Si cette mise en scène a depuis disparu, le palais de la Découverte continue néanmoins de mettre en avant une conception de la communication scientifique en adéquation avec l'ambition d'origine de donner à voir " la science en train de se faire ", avec le but ultime d'encourager les vocations scientifiques. 
Enfin, lorsque le CNRS est créé en 1939, il absorbe plusieurs institutions existantes, dont l'Office national des recherches scientifiques et industrielles et des inventions et son service de photographie et cinématographie techniques, que Jean Comandon dirigeait depuis 1920. Ce service était chargé à la fois d'assister les scientifiques dans l'utilisation du cinématographe dans leurs recherches, et de produire une documentation filmée de la recherche effectuée par l'organisme. La mission de documentation visuelle des recherches est aujourd'hui assurée par le service CNRS Images qui est aussi responsable de la conservation, l'archivage et la diffusion de ces images fixes et animées. Faut-il alors s'étonner que parmi les documents les plus anciens de ce fonds se trouvent les films réalisés par et pour Perrin sur le mouvement brownien ? Récemment mis en ligne, ils continuent, sous de nouvelles modalités technologiques, d'assurer la présence médiatique de Perrin et de sa réalité moléculaire ${ }^{15}$.

\section{Charlotte Bigg}

\section{Notes}

1 - Charlotte Bigg, Jochen Hennig (éd.) : Atombilder: Ikonographie des Atoms in Wissenschaft und Öffentlichkeit des 20. Jahrhunderts, Göttingen, 2009

2 - Christoph Lüthy, The invention of atomist iconography, in Wolfgang Lefèvre, Jürgen Renn, Urs Schöpflin (éd.) : The power of images in early modern science, Bâle, 2003, p. 117-138

3 - Christoph Meinel, Molecules and croquet balls, in Soraya de Chadarevian, Nick Hopwood (éd.) : Models. The third dimension of science, Stanford, 2004, p. 242-276

4 - Arne Schirrmacher, Looking into (the) matter : scientific artifacts and atomistic iconography, in Peter Morris, Klaus Staubermann (éd.) : Illuminating instruments, Washington, 2010, p. 131-155

5 - Jean Perrin, Mouvement brownien et réalité moléculaire, in Annales de chimie et de physique, sér. 8, vol. 8, 1909, p. 1-114, citation p. 6-7

6 - Jean Perrin, Agitation moléculaire et mouvement brownien, in Comptes rendus de l'Académie des sciences, 146, 1908, p. 967-970, citation p. 970

7 - Jean Perrin, Traité de chimie physique. Les principes, Paris, 1903, p. VII-IX

8 - Jean Perrin, Mouvement brownien et réalité moléculaire, in Annales de chimie et de physique, sér. 8, vol. 8, 1909, p. 1-114, citation p. 15

9 - Lettre du mathématicien suédois Gösta Mittag-Leffler à Henri Poincaré datée du 13 mars 1909. Philippe Narbonnaud (éd.) : La correspondance entre Henri Poincaré et Gösta Mittag-Leffler, Bâle, 1999, p. 243. Il faut mentionner aussi d'autres réactions qui se focalisent davantage sur les déterminations numériques, telles que celle du mathématicien Emile Picard : "Si les choses existent quand on peut leur faire correspondre des nombres, il n'y a plus à douter de l'existence des molécules ". Lettre à Jean Perrin datée du 30 novembre 1909, Archives de l'Académie des sciences.

10 - Voir Thierry Lefebvre, Contribution à l'histoire de la microcinématographie : de François-Franck à Comandon, in 1895, vol. 14, 1993, p. 20-36

11 - Béatrice de Pastre et Thierry Lefebvre (éd.) : Filmer la science, comprendre la vie. Le cinéma de Jean Comandon, Paris, 2012

12 - Voir à ce propos les travaux d'Oliver Gaycken, dont : Oliver Gaycken, The cinema of the future: visions of the medium as modern educator, 1895-1910, in Devin Orgeron, Marsha Orgeron, Dan Streible (éd.) : Learning with the lights off : Educational film in the United States, Oxford, 2012, p. 67-89

13 - Rapport général de la commission extra-parlementaire chargée d'étudier les moyens de généraliser l'application du cinématographe dans les différentes branches de l'enseignement, Paris, 1920, p. 4

14 - Jean Perrin, préface, in Exposition internationale. Palais de la Découverte, Paris, 1937, p. 6 